\title{
Ectopic splenic autotransplantation following traumatic injury: A case report
}

\author{
JINHE ZHANG ${ }^{1}$, JILIN YIN ${ }^{1}$, XINLU WANG $^{1}$, YINGCHEN LING $^{2}$ and JIANGTAO QUAN ${ }^{1}$ \\ ${ }^{1}$ Department of Nuclear Medicine, Guangzhou General Hospital of Guangzhou Military Command; \\ ${ }^{2}$ School of Bioscience and Bioengineering, South China University of Technology, \\ Guangzhou, Guangdong 510010, P.R. China
}

Received September 22, 2014; Accepted June 29, 2015

DOI: $10.3892 /$ etm.2015.2732

\begin{abstract}
A 41-year-old male patient was admitted to the General Hospital of Guangzhou Military Command due to upper abdominal pain persisting for $12 \mathrm{~h}$. Computed tomography $(\mathrm{CT})$ and positron emission tomography/CT scans revealed multiple soft-tissue shadows in the abdominal cavity, peritoneum and Glisson's capsule, but the metabolic activity was at normal levels. A small area of low-density shadows near the tail of the pancreas and multiple shadows of enlarged lymph nodes were identified around the porta hepatis and the pancreas, with a mildly increased metabolic activity. On the basis of the CT images the patient was diagnosed with pancreatitis. Radionuclide imaging showed the absence of the spleen from its normal position (following splenectomy), but abnormal phagocytosis of multiple red blood cells was observed in the abdomen, which was diagnosed as ectopic splenic autotransplantation (ESAT). The patient subsequently recovered well following symptomatic treatment. ESAT in trauma patients requires urgent surgery in order to remove the damaged spleen and artificially cultivate partial splenic tissue.
\end{abstract}

\section{Introduction}

Ectopic splenic autotransplantation (ESAT) following splenectomy or trauma may occasionally be encountered in clinical practice; however, clinicians or general radiology practitioners often find the diagnosis of this condition challenging (1-4). The majority of ESAT cases have no clinical symptoms and it is difficult to diagnose the exact incidence rate. Computed tomography (CT), ultrasound or magnetic

Correspondence to: Dr Jinhe Zhang or Dr Jilin Yin, Department of Nuclear Medicine, Guangzhou General Hospital of Guangzhou Military Command, 111 Liuhua Road, Guangzhou, Guangdong 510010, P.R. China

E-mail: jhjlcn@126.com

E-mail: jilinyin@yeah.net

Key words: spleen transplantation, wandering spleen, nuclide imaging resonance imaging are not specifically designed for the diagnosis of ESAT, and certain patients are misdiagnosed and undergo surgery. However, radionuclide spleen imaging has the potential of being a more specific diagnostic method for ESAT and may avert surgery. The present study reports a case of ESAT, which was diagnosed using radionuclide splenic imaging. Written informed consent was obtained from all participants.

\section{Case report}

A 41-year-old male patient was admitted to the General Hospital of Guangzhou Military Command (Guangzhou, China) due to upper abdominal pain persisting for $12 \mathrm{~h}$. The patient had been involved in a traffic collision and had undergone a splenic resection 10 years prior, which were hypothesized to have been associated with the symptoms. Physical examination revealed a $15-\mathrm{cm}$ longitudinal and transverse operational incision scar, abdominal tenderness and rebound tenderness, with a negative Murphy's sign.

Laboratory examination. The laboratory examination results were as follows: White blood cell count, $2.1 \times 10^{10}$ cells/1; neutrophils, 84.6\%; serum amylase, $246 \mathrm{U} / 1$; serum lipase, $407 \mathrm{U} / 1$; carcinoembryonic antigen, $2.27 \mathrm{~g} / \mathrm{ml}$; $\alpha$-fetoprotein, $6.17 \mathrm{mg} / \mathrm{l}$; and CA199, $22.47 \mathrm{U} / \mathrm{ml}$. These results were within normal ranges, indicating that it was unlikely that the patient's symptoms were tumor-related.

Positron emission tomography (PET)/CT scanning. The CT scan showed that the patient suffered from acute pancreatitis, severe fatty liver and small gallstones, and there were foci in S3 and S6 of the liver and multiple space-occupying foci in the ascending colon. The PET/CT scan showed the following: i) Multiple soft-tissue shadows in the abdominal cavity, peritoneum and Glisson's capsule, which were suggested to be benign lesions, since no metabolic disorder was observed; ii) a small area of low-density shadows near the tail of the pancreas, which was considered to be inflammation, since the metabolic activity was mildly increased in that area; iii) multiple shadows of enlarged lymph nodes around the porta hepatis and pancreas, with a mildly increased metabolic activity; iv) absence of the spleen, since splenectomy had been performed (Fig. 1). 

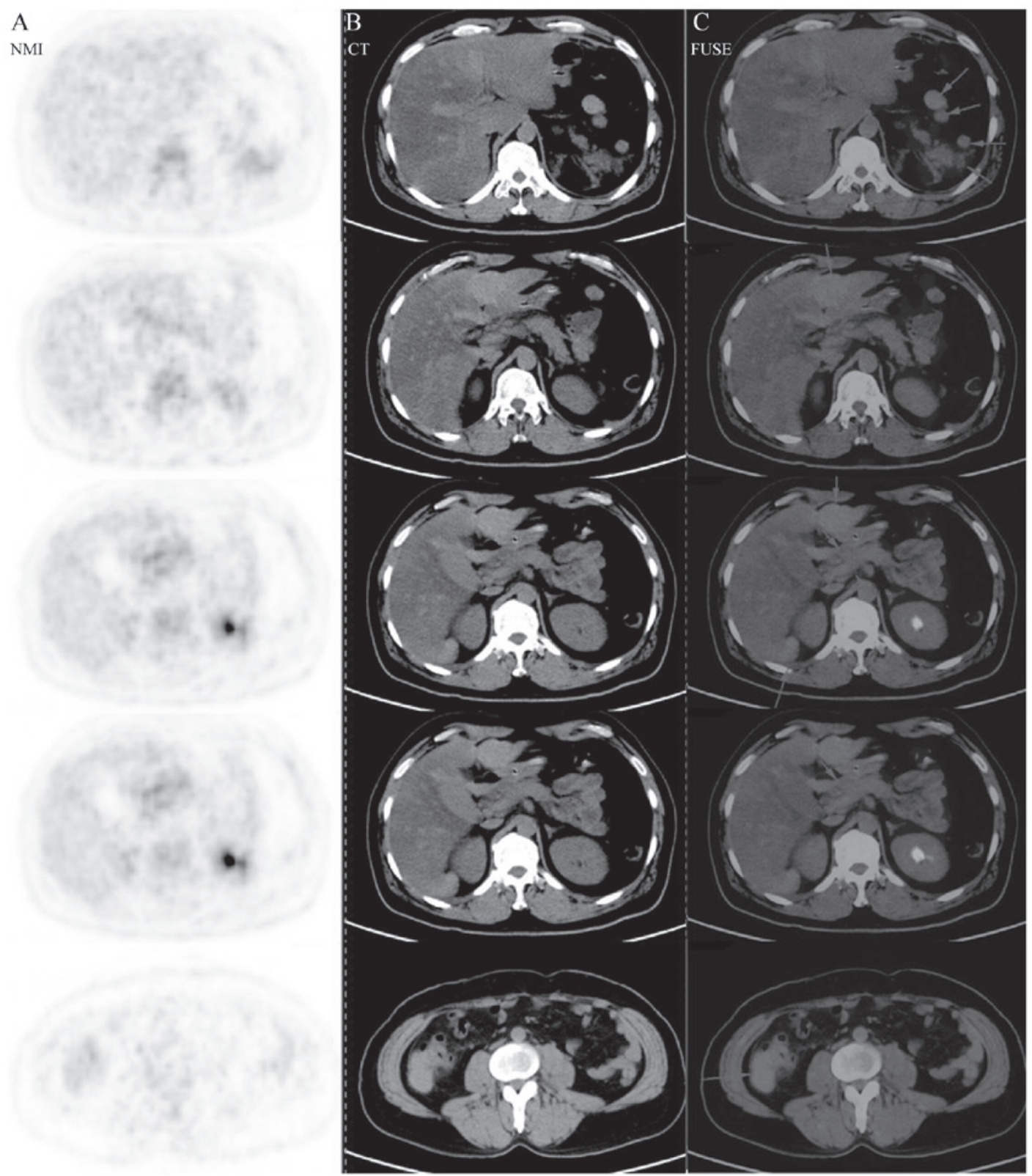

Figure 1. Positron emission tomography/computed tomography (CT) scanning revealed multiple soft-tissue shadows in the abdominal cavity, peritoneum and Glisson's capsule (arrows in the FUSE images). (A) Nuclear medicine imaging (NMI) indicated normal metabolic activity. (B and C) A small area of low-density shadows was observed near the tail of pancreas. As the metabolic activity was mildly increased in the area, it was considered to be inflammation. Multiple shadows of enlarged lymph nodes were observed around the porta hepatis and pancreas, with a mildly increased metabolic activity.

A

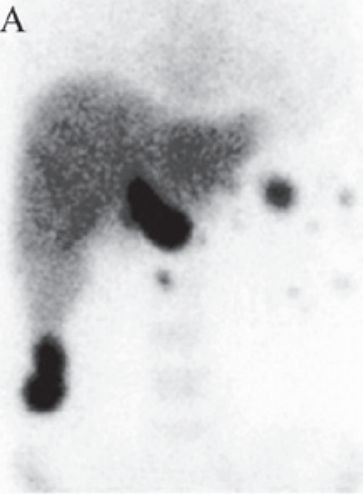

B

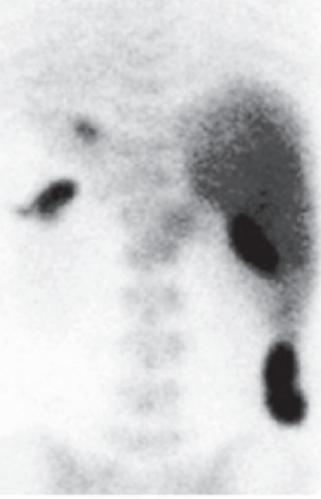

$\mathrm{C}$

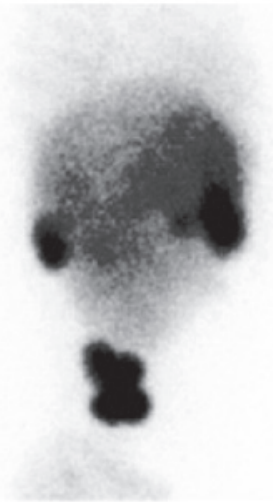

$\mathrm{D}$

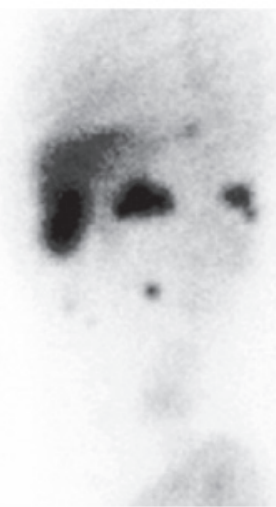

Figure 2. Planar images at the (A) anteroposterior, (B) posteroanterior, (C) right lateral and (D) left lateral position 30 min following intravenous injection of ${ }^{99 \mathrm{~m}} \mathrm{Tc}-$ labeled heat-denatured erythrocytes. Absence of the spleen from its normal position (following splenectomy) was observed. Multiple areas with increased radioactivity were irregularly distributed in the abdomen, which was diagnosed as ectopic splenic autotransplantation. 
Radionuclide imaging. The spleen was absent from its normal position (following splenectomy), but abnormal phagocytosis of multiple red blood cells was observed in the abdomen, which was considered to be ESAT (Fig. 2).

Clinical diagnosis. The patient was diagnosed with acute pancreatitis, cholecystitis with gallstones, fatty liver and ESAT following splenectomy. The patient subsequently recovered well after receiving antipyretic analgesics and rehydration therapy.

\section{Discussion}

ESAT refers to the spontaneous transplantation of splenic tissue following injury or splenectomy. ESAT may occur in various sites, such as the chest, pelvic cavity or subcutaneous tissue, but is mainly found in the abdominal cavity (5-7). In the majority of cases, ESAT is asymptomatic and does not affect any physiological function; therefore, intervention is usually not required (8). In the present case, the patient presented with acute pancreatitis and ESAT was detected accidentally; it did, however, affect clinical diagnosis to a great extent, as ESAT is easily misdiagnosed as a tumor. Conventional radionuclide splenic imaging is a method specific for the diagnosis of ESAT, and in the present case it proved to be more diagnostically significant compared with PET/CT.

\section{References}

1. Oda J, Tachikawa N and Suda T: A solid mass in pelvic region. Gastroenterology 137: e9-e10, 2009.

2. Wallace S, Herer E, Kiraly J, Valikangas E and Rahmani R: A wandering spleen: Unusual cause of a pelvic mass. Obstet Gynecol 112: 478-480, 2008.

3. Fiquet-Francois C, Belouadah M, Ludot $\mathrm{H}$, et al: Wandering spleen in children: Multicenter retrospective study. J Pediatr Surg 45: 1519-1524, 2010.

4. Bouassida M, Sassi S, Chtourou MF, et al: A wandering spleen presenting as a hypogastric mass: Case report. Pan Afr Med J 11: 31, 2012.

5. Di Crosta I,Inserra A, Gil CP,Pisani M and Ponticelli A: Abdominal pain and wandering spleen in young children: The importance of an early diagnosis. J Pediatr Surg 44: 1446-1449, 2009.

6. Dirican A, Burak I, Ara C, Unal B, Ozgor D and Meydanli MM: Torsion of wandering spleen. Bratisl Lek Listy 110: 723-725, 2009.

7. Tseng CA and Chou AL: Images in clinical medicine. Pelvic spleen. N Engl J Med 361: 1291, 2009.

8. Dillman JR and Strouse PJ: Clinical image. The 'wandering' spleen. Pediatr Radiol 40: 231, 2010. 